\title{
Preparation, Microstructural Characterization, and Selected Mechanical Properties of Ti-20Zr-2.5Mo and Ti-20Zr-7.5Mo used as Biomaterial
}

\author{
Pedro Akira Bazaglia Kuroda, 1,2,a; Marília Afonso Rabelo Buzalaf ${ }^{3, b}$ \\ and Carlos Roberto Grandini i, ${ }^{1, c}$
}

\author{
${ }^{1}$ UNESP - Univ. Estadual Paulista, Laboratório de Anelasticidade e Biomateriais, 17.033.360, \\ Bauru, SP, Brazil \\ ${ }^{2}$ IBTN - Institute of Biomaterials, Tribocorrosion and Nanomedicine \\ ${ }^{3}$ USP - Univ. São Paulo, Departamento de Ciências Biológicas e Bioquímica, 17.012-901, \\ Bauru, SP, Brazil

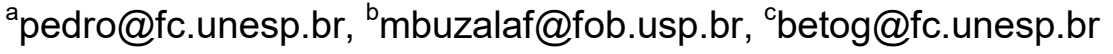

Keywords: Titanium alloys, microstructure, biomaterial.

\begin{abstract}
Titanium is used in the biomedical field due to its mechanical strength/density, corrosion resistance, and biocompatibility. In this paper, the preparation, and the structural, microstructural, and mechanical characterization of $\mathrm{Ti}-20 \mathrm{Zr}-2.5 \mathrm{Mo}$ and $\mathrm{Ti}-20 \mathrm{Zr}-7.5 \mathrm{Mo}$ alloys are presented. The elements were melted into an arc furnace with an argon controlled atmosphere. To determine the amount of impurities present in each alloy, an analysis of the chemical composition was conducted using EDS. The samples were characterized by measurements of density, X-ray diffraction (with the diffractograms refined by the Rietveld method), and optical and scanning electron microscopy. The mechanical properties were evaluated using Vickers microhardness test and modulus of elasticity. The results showed that that $\alpha / \alpha "$ and $\alpha \prime / \beta$ phases coexisted in both of the prepared alloys, respectively. The alloys have higher hardness than $\mathrm{cp}-\mathrm{Ti}$ and their modulus of elasticity values are very close to the modulus values of cp-Ti.
\end{abstract}

\section{Introduction}

Titanium and its alloys are used in the biomedical field due to their favorable characteristics, including good mechanical strength/density ratio, high corrosion resistance, and excellent biocompatibility [1,2]. Titanium is a transition metal that undergoes an allotropic transformation around $883^{\circ} \mathrm{C}$. Below this temperature, its crystalline structure is hexagonal compact ( $\alpha$ phase). Above this temperature, it has a cubic body-centered cubic crystalline structure ( $\beta$ phase), which remains stable until metal fusion occurs [1]. Zirconium undergoes an allotropic transformation similar to titanium at around $862^{\circ} \mathrm{C}$. It is considered to be a neutral element that can be used to form titanium alloys [3]. Molybdenum has a body-centered cubic crystalline structure. The addition of molybdenum decreases the phase change temperature of titanium ( $\beta$-transus), and it is considered to be a strong $\beta$-stabilizer, with the $\beta$ phase retention starting at around $10 \%$ in weight [4]. Titanium can present metastable phases, which are formed out of equilibrium and which prevent the rearrangement of atoms by means of atomic diffusion or mechanical strain. These are the martensitic $\alpha$ ' (distorted hexagonal crystalline structure) and $\alpha$ " (orthorhombic structure) phases and the $\omega$ (trigonal or hexagonal structure) phases [5,6].

In the present study, the structure, microstructure, and some of the properties of $\mathrm{Ti}-20 \mathrm{Zr}-2.5 \mathrm{Mo}$ and Ti-20Zr-7.5Mo alloys were investigated.

\section{Materials and Methods}

In this study, cp-Ti (ASTM F67), cp-Zr (99.8\% purity), and cp-Mo (99.9\% purity) were used as precursor materials. The melting was performed using an arc-voltaic furnace with a water cooled copper crucible, a non-consumable tungsten electrode, and a controlled argon atmosphere. The alloys were melted with $20 \%$ in weight of zirconium and $2.5 \%$ and $7.5 \%$ in weight of molybdenum. 
To verify the stoichiometry and homogeneity of the alloys, chemical analysis and a mapping of the distribution of the components of the alloys were performed using energy-dispersive spectroscopy X-ray (EDS) (Oxford, INCA model).

To confirm the stoichiometry of the produced alloys, density measurements were made using Archimedes' principle and an Ohaus analytical balance (Explorer model).

The structure was analyzed by means of X-ray diffraction measurements; quantitative analysis of the phases and the calculation of the lattice parameters were performed using the Rietveld method. $\mathrm{X}$-ray diffraction measurements were obtained using the powder method in a Rigaku diffractometer (D/Max-2100PC model), with $\mathrm{Cu}-\mathrm{K} \alpha$ radiation $(\lambda=1.544 \AA$ ), $20 \mathrm{~mA}$ current, potential of $40 \mathrm{kV}$, permanence time of $3.2 \mathrm{~s}$, and step of 0.02 degree, in the range of $10^{\circ}$ to $100^{\circ}$ and fixed time mode.

To analyze the diffractograms by the Rietveld method, GSAS ${ }^{\circledR}$ software [7] and the EXPIGUI interface [8], with the crystallographic database records of the Inorganic Crystal Structure Database (ICSD), were used. The crystallographic sheets were used based on the crystalline structure of titanium compared to the diffraction peaks obtained in the alloys.

The microstructure was obtained by optical and scanning electron microscopy. For optical microscopy, an Olympus microscope (model BX51M) was used. For scanning electron microscopy, a Carl Zeiss microscope (model EVO-015) was used.

The initial verification of the mechanical properties was done using Vickers microhardness measurements. A Shimadzu microhardness tester (model HMV-2) with a load of $200 \mathrm{~g}(1.961 \mathrm{~N})$ and a penetration time of $60 \mathrm{~s}$ was used. To measure the modulus of elasticity, Sonelastic $\AA$ equipment was used, based on the impulse excitation technique.

\section{Results and Discussion}

Table 1 shows the values obtained for the components of the alloys, using EDS. The values found are very close to the values of the proposed composition, showing that the stoichiometry of the alloys was respected. For example, Figure 1 shows an image of the EDS mapping of the elements that make up a sample of the Ti-20Zr-2.5Mo alloy. A homogeneous distribution, without the presence of clusters or segregated, can be observed.

Table 1. EDS analysis of the elements that comprise the Ti20Zr-2.5Mo and Ti-20Zr-7.5Mo alloys.

\begin{tabular}{llll}
\hline Samples & Ti (wt\%) & Zr (wt\%) & Mo (wt\%) \\
\hline Ti-20Zr-2.5Mo & $76.3 \pm 0.1$ & $21.0 \pm 0.1$ & $2.6 \pm 0.1$ \\
Ti-20Zr-7.5Mo & $71.1 \pm 0.1$ & $20.9 \pm 0.1$ & $7.9 \pm 0.1$ \\
\hline
\end{tabular}

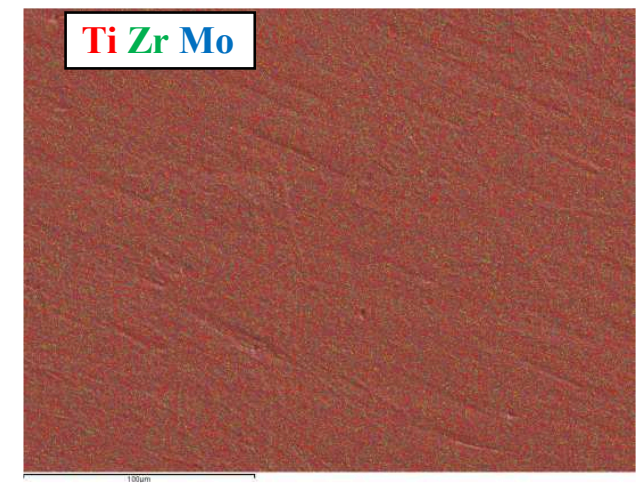

Fig. 1. EDS mapping of the Ti20Zr-2.5Mo alloy.

Figure 2 presents the density values of the Ti- 20Zr-2.5 Mo and Ti-10Zr-7.5 Mo alloys, after melting. The increase in the density with the addition of the alloying elements is related to the higher density values and the atomic weight of molybdenum $(95.94 \mathrm{~g} / \mathrm{mol} ; 10.23 \mathrm{~g} / \mathrm{cm} 3)$ and zirconium $(91.22 \mathrm{~g} / \mathrm{mol} ; 6.51 \mathrm{~g} / \mathrm{cm} 3)$ as compared to titanium $(47.88 \mathrm{~g} / \mathrm{mol} ; 4.51 \mathrm{~g} / \mathrm{cm} 3)$ [3].

Figure 3 shows a comparison of the diffraction patterns for the alloys after melting with the precursor materials. The presence of peaks, characteristics of the martensitic $\alpha$ ' phase and $\alpha$ ' phase in the alloy with $2.5 \mathrm{wt} \%$ of molybdenum were observed. The alloy with $7.5 \mathrm{wt} \%$ of molybdenum exhibited peaks characteristics of $\alpha$ " phase; however, peaks associated with the $\beta$ phase were also observed. The presence of $\alpha$ " phase in the Ti-20Zr-2.5Mo alloy and $\beta$ phase in the Ti-20Zr-7.5Mo alloy is associated with the characteristic of molybdenum when decreasing the temperature of $\beta$ 
phase transformation in titanium alloys, i.e. molybdenum is a strong $\beta$-stabilizer element and phase $\beta$ retention may be initiated when the concentration of molybdenum is between $9 \mathrm{wt} \%$ and $10 \mathrm{wt} \%$ [4]. Another indication of the appearance of these phases is that zirconium is a neutral element, but it helps in stabilizing the $\beta$ phase slightly in the presence of molybdenum, tantalum, niobium, or other $\beta$-stabilizer elements $[9,10]$.

The result of refinement of the X-ray diffractogram for the Ti-20Zr-2.5Mo alloy using the Rietveld method is shown in Figure 4. A good agreement was obtained between the experimental values and the calculated values, indicating a good refinement of the data. In Table 2, the parameters of merit for refinement of the diffractograms by the Rietveld method are presented. The results of the refinement, shown in Table 3, reinforce the coexistence of the $\alpha$ ' $+\alpha$ '" phases in the alloy with $2.5 \%$ in weight of molybdenum and the $\alpha "+\beta$ phases in the alloy with $7.5 \%$ in weight of molybdenum. The $\alpha$ phase lattice parameters were dilated with respect to the titanium due to the addition of zirconium, because that element has a larger atomic radius $(1.59 \AA)$ than titanium $(1.45 \AA)$ and the same hexagonal structure.. The addition of molybdenum does not significantly change the lattice parameters of the body-centered cubic cell because molybdenum has a smaller atomic radius (1.36 ̊́) than titanium [3].

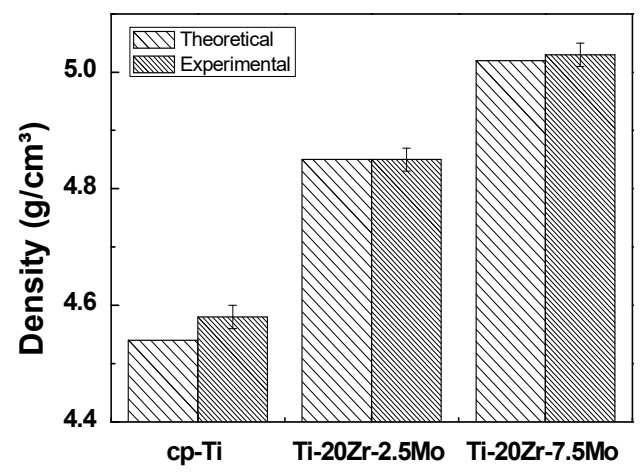

Fig. 2. Density of the Ti-20Zr-2.5Mo and Ti-10Zr-7.5Mo alloys, compared with cp-Ti.

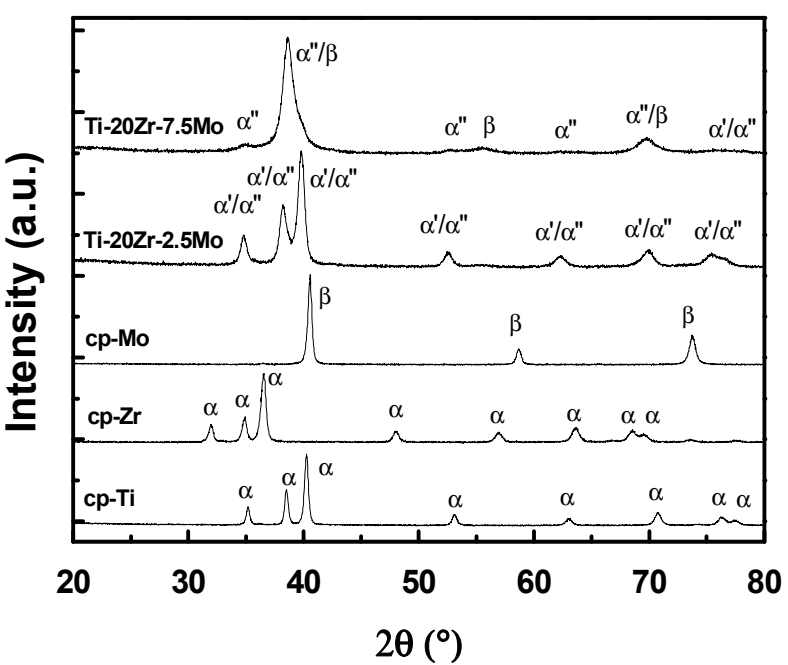

Fig. 3. X-ray diffractograms of the Ti-20Zr-2.5Mo and Ti-10Zr-7.5Mo alloys, compared with commercially pure $\mathrm{Ti}, \mathrm{Zr}$, and Mo.

Figure 5 shows the micrographs of the Ti- 20Zr-2.5Mo and Ti-20Zr-7.5Mo alloys, with magnifications of 500x and 1000x ( $\mathrm{a}$ and $\mathrm{d}, \mathrm{b}$ and $\mathrm{e}$, respectively) obtained with the optical microscope and a magnification of 3000x (c and e, respectively) obtained with the scanning electron microscope. The microstructure of the Ti-20Zr-2.5Mo alloy is composed of small acicular structures in the intragrain region, which are characteristic of the martensitic $\alpha$ ' and $\alpha$ ' phases. For the Ti-20Zr-7.5Mo alloy, a microstructure consisting of equiaxial grains from the $\beta$ phase, with a small amount of the martensitic $\alpha^{\prime}$ phase in the intragrain region, was found in the X-ray diffraction results. 


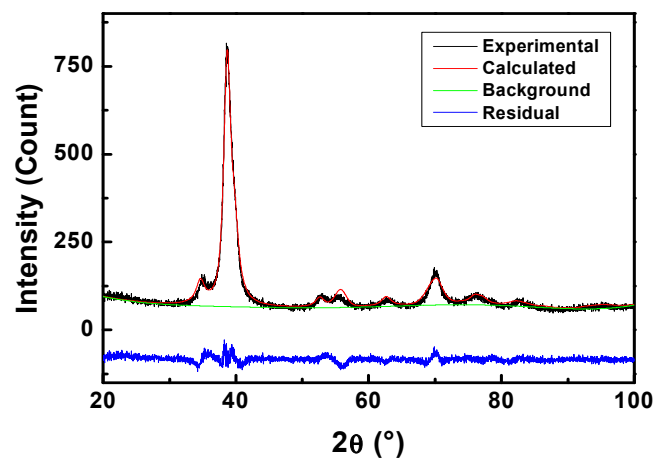

Table 2. Quality parameters obtained after refinement with the Rietveld method.

\begin{tabular}{lcc} 
Sample & $\chi^{2}$ & $\mathrm{RF}^{2}(\%)$ \\
\hline Ti-20Zr- & 1.944 & 12.01 \\
$\mathbf{2 . 5 M o}$ & 1.701 & 10.13 \\
Ti-20Zr- & & \\
7.5Mo & & \\
\hline
\end{tabular}

Fig. 4. Refinement of the X-ray diffractogram for Ti-20Zr-2.5Mo.

Table 3. Structural parameters and phase percentage for the Ti-20Zr-2.5Mo and Ti-20Zr-7.5Mo alloys.

\begin{tabular}{lccc|ccc}
\hline \multirow{2}{*}{ Sample } & \multicolumn{3}{c|}{ Phase $(\%)$} & \multicolumn{3}{c}{ Lattice parameter $(\AA)$} \\
\cline { 2 - 7 } & $\alpha^{\prime}$ & $\alpha{ }^{\prime}$ & $\beta$ & $\mathrm{a}$ & $\mathrm{b}$ & $\mathrm{c}$ \\
\hline Ti-20Zr-2.5Mo & 63.57 & 36.43 & 0 & $2.988(5)$ & $2.988(5)$ & $4.731(8)$ \\
& & & & $3.311(5)$ & $5.130(7)$ & $4.728(6)$ \\
\hline Ti-20Zr-7.5Mo & 0 & 22.78 & \multirow{2}{*}{77.22} & $3.09(7)$ & $5.04(1)$ & $4.71(1)$ \\
& & & & $3.32(1)$ & $3.32(1)$ & $3.32(1)$ \\
\hline
\end{tabular}

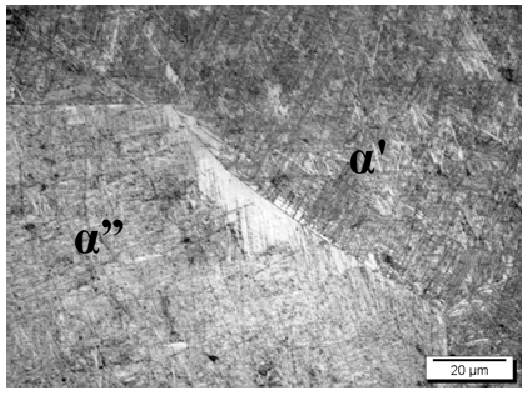

(a)

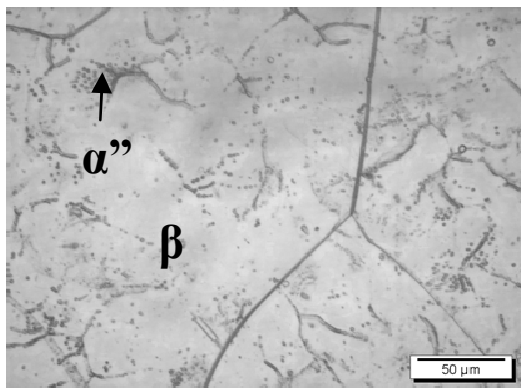

(d)

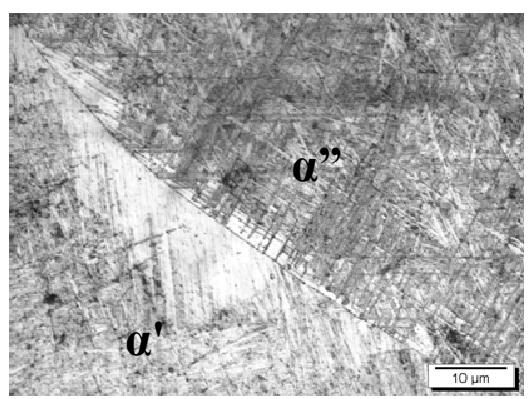

(b)

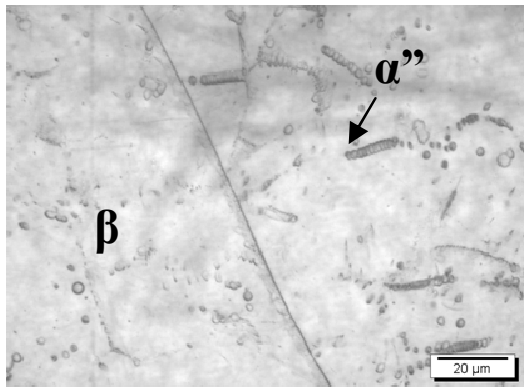

(e)

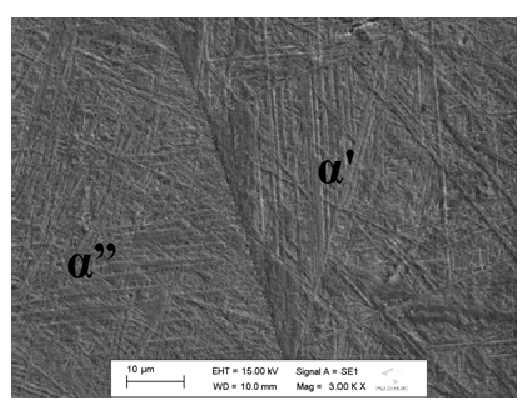

(c)

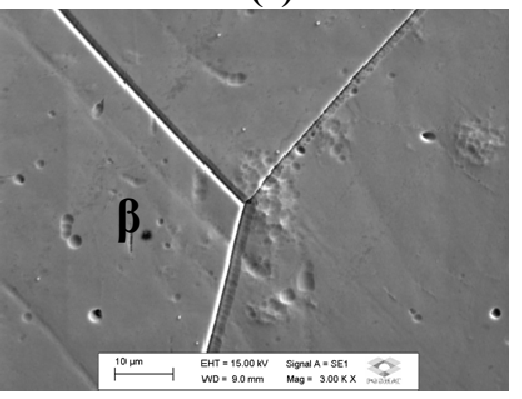

(f)

Fig. 5. Micrographs of Ti-20Zr-2.5Mo with magnifications of 500x (a), 1000x (b), and 3000x (c). Micrographs of Ti-20Zr-7.5Mo with magnifications of 500x (d), 1000x (e), and 3000x (f).

Figure 6 presents the results of the Vickers microhardness measurements for the Ti-20Zr-2.5Mo and Ti-20Zr-7.5Mo alloys. As shown, the two alloys have hardness values significantly higher than pure titanium, giving the indication that their greater mechanical resistance is the result of the hardening by the solid solution caused by the addition of zirconium and molybdenum, which have a higher atomic mass ( $\mathrm{Zr}=91.22$ u.m.a and $\mathrm{Mo}=95.94$ u.m.a) than titanium (47.90 u.m.a) [3]. 
Figure 7 shows the results of the modulus of elasticity test for the studied alloys. It also presents a comparison of the values of the mechanical properties of materials already known and used in the biomedical field. The modulus of elasticity for Ti-20Zr-2.5Mo and Ti-20Zr-7.5Mo is similar to cp$\mathrm{Ti}$ and, depending on the type of treatment applied, the alloy material can offer more advantageous features than titanium, showing good potential for use as an orthopedic implant.

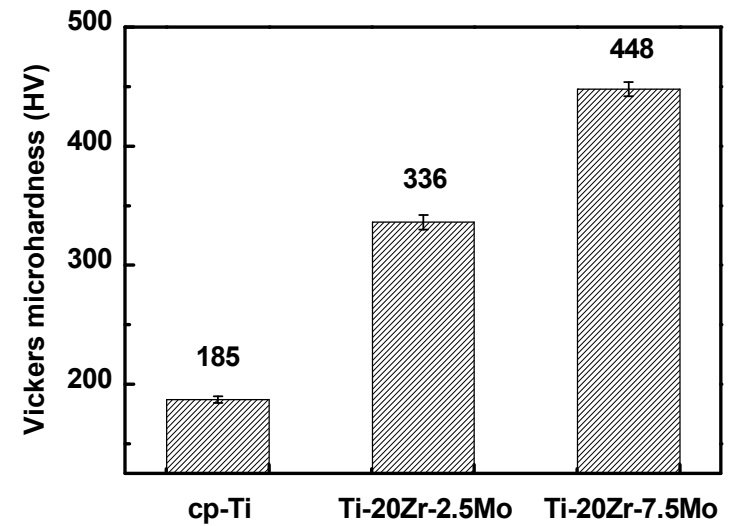

Fig. 6. Vickers microhardness for the Ti20Zr-2.5Mo and Ti-10Zr-7.5Mo alloys, compared with cp-Ti.

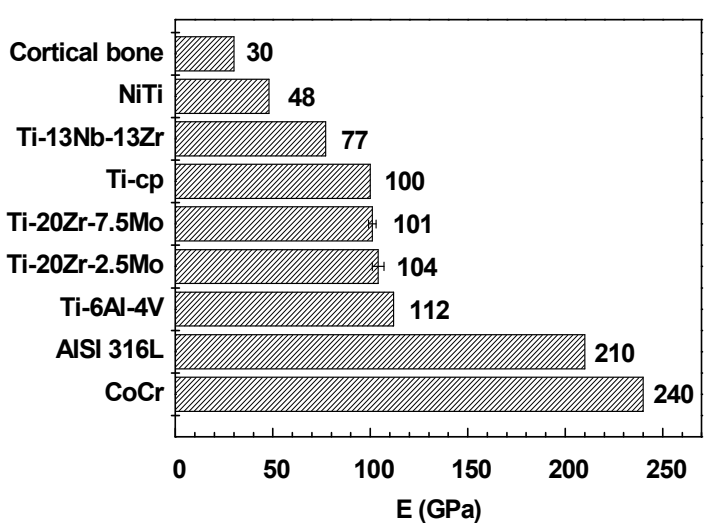

Fig. 7. Young's modulus of elasticity for the Ti20Zr-2.5Mo and Ti-10Zr-7.5Mo alloys, compared with commercial metals used as biomaterial.

\section{Conclusions}

From the results presented in this paper it is possible to conclude that the melting of the alloys was successful, producing fine quality materials, as shown by the chemical composition analysis, mapping, and density measurements. The results of the structural and microstructural analysis of the alloys showed that the microstructure of the Ti-20Zr-2.5Mo alloy is formed by the martensitic $\alpha$ ' and $\alpha$ " phases. The $\alpha$ " and $\beta$ phases are already present in the Ti-20Zr-7.5Mo alloy. The hardness of the alloys was higher than the hardness of commercially pure titanium, due to the solid solution hardening of the alloys. The modulus of elasticity of the studied alloys is similar to the modulus of elasticity of cp-Ti.

\section{Acknowledgements}

The authors thank the following Brazilian agencies for their financial support: Capes, CNPq (grants \#481313/2012-5 and \#307.279/2013-8) and FAPESP (grant \# 2012/22.742-6).

\section{References}

[1] C. M. Lee, C. P. Ju and J. H. Lin: Journal of Oral Rehabilitation Vol. 29 (2002), p. 314.

[2] M. Geetha, A.K. Singh, R. Asokamani and A.K. Gogia: Progress in Materials Science Vol. 54 (2009), p. 397.

[3] D. Lide: CRC handbook of chemistry and physics: a ready-reference book of chemical and physical data, 85th, CRC Press, Boca Raton, USA, 2004.

[4] W.F. Ho, C.P. Ju and J.H. Chern Lin: Biomaterials Vol. 20 (1999), p. 2115.

[5] C. Leyens and M. Peters: Titanium and Titanium Alloys: Fundamentals And Applications. (Wiley-VCH, New York, 2005).

[6] S. Nag, R. Banerjee and H. Fraser: J Mater Sci Vol. 44 (2009), p. 808.

[7] A.C. Larson, R.B. Von Dreele, "General Structure Analysis System (GSAS)", Los Alamos National Laboratory Report LAUR (1994) 86-748 
[8] B. Tob: Journal of Applied Crystallography Vol. 34 (2001), p. 210.

[9] W.F. Ho, S.C. Wu, S.K. Hsu, Y.C. Li and H.C. Hsu: Materials Science and Engineering C Vol. 32 (2012), p. 517.

[10] D.R.N. Correa, P.A.B. Kuroda and C.R. Grandini: Advanced Materials Research Vol. 922 (2014), p. 75. 


\section{1st Brazilian Conference on Materials Science and Engineering}

10.4028/www.scientific.net/MSF.869

Preparation, Microstructural Characterization, and Selected Mechanical Properties of Ti-20Zr-2.5Mo and Ti-20Zr-7.5Mo Used as Biomaterial

10.4028/www.scientific.net/MSF.869.946

\section{DOI References}

[1] C. M. Lee, C. P. Ju and J. H. Lin: Journal of Oral Rehabilitation Vol. 29 (2002), p.314.

10.1046/j.1365-2842.2002.00825.x

[2] M. Geetha, A.K. Singh, R. Asokamani and A.K. Gogia: Progress in Materials Science Vol. 54 (2009), p.397.

10.1016/j.pmatsci.2008.06.004

[3] D. Lide: CRC handbook of chemistry and physics: a ready-reference book of chemical and physical data, 85th, CRC Press, Boca Raton, USA, (2004).

$10.1021 / \mathrm{ja} 041017 \mathrm{a}$

[4] W.F. Ho, C.P. Ju and J.H. Chern Lin: Biomaterials Vol. 20 (1999), p.2115.

10.1016/s0142-9612(99)00114-3

[5] C. Leyens and M. Peters: Titanium and Titanium Alloys: Fundamentals And Applications. (Wiley-VCH, New York, 2005).

10.1002/3527602119.ch8

[6] S. Nag, R. Banerjee and H. Fraser: J Mater Sci Vol. 44 (2009), p.808.

$10.1007 / \mathrm{s} 10853-008-3148-2$

[9] W.F. Ho, S.C. Wu, S.K. Hsu, Y.C. Li and H.C. Hsu: Materials Science and Engineering C Vol. 32 (2012), p.517.

10.1016/j.msec.2011.12.003

[10] D.R.N. Correa, P.A.B. Kuroda and C.R. Grandini: Advanced Materials Research Vol. 922 (2014), p.75. 10.4028/www.scientific.net/amr.922.75 
Reproduced with permission of the copyright owner. Further reproduction prohibited without permission. 